\title{
Modeling and Analysis of Chaotic Behavior in Switched Reluctance Motor Drives
}

\author{
J.H. Chen, K.T. Chau, Q. Jiang, C.C. Chan and S.Z. Jiang \\ Dept. of Electrical \& Electronic Engineering, University of Hong Kong, Hong Kong, China
}

\begin{abstract}
In this paper, modeling and analysis of chaotic behavior in switched reluctance (SR) motor drives using voltage PWM regulation is presented. The key is to derive a Poincaré map that is based on the nonlinear flux linkage model. Its Jacobian matrix can be evaluated by solving the corresponding variational equation. Based on the Poincaré map and its Jacobian matrix, the analysis of chaotic behavior is presented. Furthermore, bifurcation diagrams are also figured out. They facilitate to determine the stable range of various system parameters so as to avoid the occurrence of chaos. Both computer simulations and experimental measurements are given to verify the theoretical modeling and analysis.
\end{abstract}

\section{INTRODUCTION}

In recent years, switched reluctance (SR) motor drives have been recognized to have promising industrial applications. Their simplicity in both motor construction and power converter requirement offers the prospect of low-cost fully controllable brushless motor drives [1]. Recently, the research on SR motor drives has been focused on motor design, converter topology and control strategy. However, the investigation into the nonlinear dynamics, namely chaotic behavior, of SR motor drives has been surprisingly rare [2]. The major reason should be due to the complexity of analytical formulation and high nonlinearities of SR motor drives.

Chaos is a common phenomenon in power converters when they operate under feedback control [3]-[4]. Chaotic behavior in switching mode power supplies has been actively investigated for a number of years. Chaos in induction and brushless de motor drives has also been discussed [5]-[6]. Very recently, the nonlinear dynamics and chaotic behavior of industrial dc motor drives have been investigated, by using numerical simulation and analytical modeling [7]. It has been identified that the nonlinear dynamics of the de motor drive system is mainly due to the switching nonlinearity caused by voltage PWM regulation. Although the SR motor drive using voltage PWM regulation operates like the dc motor drive, its nonlinear dynamics are much more complicated, hence more prone to chaos.

The purpose of this paper is to develop the modeling of chaos in the SR motor drive, which includes the formulation of the system modeling, the derivation of the Poincare map and its Jacobian matrix. Based on the Poincaré map and its Jacobian matrix, the analyses of periodic solutions and chaotic behavior are also discussed. In order to attain the insight of chaotic behavior, both time-domain waveforms and phaseplane trajectories are investigated. Moreover, bifurcation diagrams are also figured out so as to determine stable regions of the system parameters.
In Section II, the system modeling of the SR motor drive using the voltage PWM regulation is presented. Then, the Poincaré map and its Jacobian matrix are derived in Section III. Analysis of chaotic behavior and computer simulations are respectively discussed in Sections IV and V. Finally, experimental measurements are given to verify the theoretical modeling and analysis in Section VI.

\section{MODELING OF SR MOTOR DRIVES}

As shown in Fig. 1, an adjustable speed $m$-phase SR motor drive is used for exemplification. The corresponding speed control is achieved by applying PWM chopping of its three-phase motor voltages. The commutation logic uses rotor position feedback to select the turn-on angle $\theta_{o}$ and turn-off angle $\theta_{c}$ of those lower-leg power switches $\left(A_{2}, B_{2}\right.$ and $C_{2}$ ). In order to simplify the control circuit, the dwell angle $\theta_{d}=\theta_{c}-\theta_{0}$ of each phase winding is selected to be equal to the commutation angle $\theta_{s}=2 \pi /\left(m N_{r}\right)$, where $N_{r}$. is the number of rotor poles.

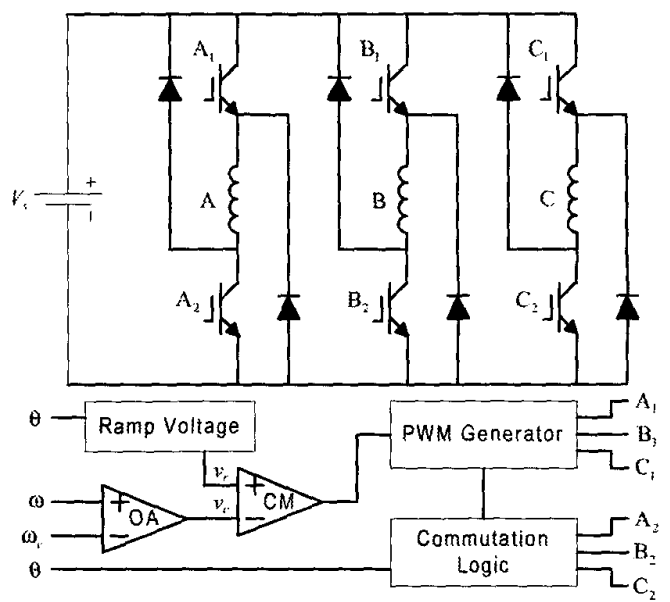

Fig. 1. Schematic diagram of SR motor drive

For the sake of synchronizing the voltage PWM regulation with the phase commutation, the PWM carrier signal ramp voltage $v_{r}$ for each phase winding is a function of the instantaneous rotor displacement $\theta$ :

$v_{r}=v_{i}+\left(v_{u}-v_{l}\right)\left[\left(\theta-\theta_{o}\right) \bmod \theta_{r}\right] / \theta_{T}$

where $v_{l}$ and $v_{u}$ are the lower and upper bounds of the ramp voltage, $\theta_{T}=\theta_{d} / n_{\theta}$ is its period, and $n_{\theta}$ is an integer. As the op-amplifier OA has a feedback gain $g$, the speed control signal $v_{c}$ can be expressed as: 
$v_{c}=g\left(\omega-\omega_{c}\right)$

where $\omega$ and $\omega_{c}$ are the instantaneous and command speed of the SR motor drive. Then, both $v_{r}$ and $v_{c}$ are fed into the comparator $\mathrm{CM}$ which outputs the signal to turn on or off those upper-leg power switches $\left(A_{1}, B_{1}\right.$ and $\left.C_{1}\right)$, depending on the phase commutation. When the control voltage exceeds the ramp voltage, the upper-leg switch being the same phase of the turn-on lower-leg switch is off; otherwise it is on. The other phase switches remain off.

Since the dwell angle is equal to the commutation angle, only two adjacent phase windings have currents at the same time. For the sake of clarity and simplicity, $m$ phase windings conducted in turn are represented by two virtual phase windings activated alternatively. When the winding is conducted, it is called the activated winding; otherwise it is called the inactivated winding. Thus, considering the winding 2 lags behind the winding 1 in the commutation angle $\theta_{s}$, the system equation of the SR motor drive is given by:

$$
\left\{\begin{array}{l}
\frac{d \theta}{d t}=\omega \\
\frac{d \omega}{d t}=\left(-B \omega+T_{v}\left(\theta, \Psi_{1}, \psi_{2}, \theta_{s}\right)-T_{1}\right) / J \\
\frac{d \psi_{1}}{d t}=-R i_{1}\left(\theta, \Psi_{1}\right)+u_{1}(\theta) \\
\frac{d \psi_{2}}{d t}=-R i_{2}\left(\theta-\theta_{s}, \psi_{2}\right)+u_{2}\left(\theta-\theta_{s}\right)
\end{array}\right.
$$

$T_{c}=\sum_{k=1}^{m}\left(-\frac{\partial}{\partial \theta} \int_{0}^{\psi_{k}} i_{k}\left(\theta-(k-1) \theta_{s}, \psi_{k}\right) d \psi\right)$

$u_{k}=\left\{\begin{array}{lr}V_{s} \varepsilon\left(v_{r}(\theta)-v_{c}\right) \quad\left(\theta \bmod 2 \theta_{s}\right) \in\left[\theta_{o}, \theta_{o}+\theta_{s}\right) \\ -V_{s} \varepsilon\left(\psi_{k}\right) \quad\left(\theta \bmod 2 \theta_{s}\right) \in\left[\theta_{o}+\theta_{s}, \theta_{o}+2 \theta_{s}\right)\end{array}\right.$

where $u_{k}$ is the phase voltage, $i_{k}$ is the phase current, $\psi_{k}$ is the phase flux linkage, $R$ is the phase resistance, $L$ is the phase inductance, $B$ is the viscous damping, $J$ is the load inertia, $T_{l}$ is the load torque, and $\varepsilon$ is a unit step function. $i_{k}$ is expressed by an interpolation function of $\theta$ and $\psi_{k}$.

By defining the state vector $X=\left(\theta, \omega, \psi_{1}, \psi_{2}\right)^{\mathrm{T}}$ and the output vector $\mathrm{Y}=(\theta, \omega, i)$, where $i=i_{1}+i_{2}$, (3) can be rewritten as:

$\left\{\begin{array}{l}\dot{\mathbf{X}}=f_{i}(\mathbf{X}) \\ \mathbf{Y}=M(\mathbf{X})\end{array}\right.$

1II. POINCARÉ MAP AND ITS JACOBIAN MATRIX In order to derive the Poincaré map, a plane is defined as: $\Sigma:=\left\{\mathbf{X}:\left[\left(\theta-\theta_{o}\right) \bmod \theta_{s}\right]=0\right\}$

The trajectory of $\mathbf{X}$ under observation repeatedly passes through the $\Sigma$ when $\theta$ increases monotonically. The sequence of $\Sigma$ crossing defines the Poincare map:
$P: \Re^{3} \rightarrow \Re^{3},\left(\omega, \psi_{1}, \psi_{2}\right)_{n+1}^{\mathrm{T}}=P\left(\left(\omega, \psi_{3}, \psi_{2}\right)_{n}^{\mathrm{T}}\right)$

Actually, the orbit of this map is a sequence of samples at the beginning of the dwell angle of each phase winding. In order to avoid the calculation of the plane crossing, the rotor displacement $\theta$, rather than time $t$, is selected as the independent variable of the system. The next crossing of the plane $\theta_{n+1}=\theta_{o}+(n+1) \theta_{s}$ can be directly calculated by integrating from $\theta_{n}=\theta_{0}+n \theta_{s}$ to $\theta_{n+1}$. To make $\theta$ as an independent variable, (3) is expressed as:

$$
\left\{\begin{aligned}
\dot{\omega} & =\left(-B \omega+T_{i}\left(\theta, \psi_{1}, \psi_{2}, \theta_{s}\right)-T_{L}\right) /(J \omega) \\
\dot{\psi}_{1} & =\left(-R i_{1}\left(\theta, \psi_{1}\right)+u_{1}(\theta)\right) / \omega \\
\dot{\psi}_{2} & =\left(-R i_{2}\left(\theta-\theta_{s}, \psi_{2}\right)+u_{2}\left(\theta-\theta_{s}\right)\right) / \omega
\end{aligned}\right.
$$

By redefining the state vector $\mathbf{X}(\theta)=\left(\omega, \psi_{1}, \psi_{2}\right)^{\mathrm{T}}$ and output vector $\mathbf{Y}(\theta)=(\omega, i)^{\Upsilon},(9)$ can be rewritten as:

$$
\left\{\begin{array}{l}
\dot{\mathbf{X}}=f_{\theta}(\mathbf{X}, \theta) \\
\mathbf{Y}=M(\mathbf{X})
\end{array}\right.
$$

The Poincaré map (8) can also be rewritten as:

$\left\{\begin{aligned} \mathbf{X}_{n+1} & =P\left(\mathbf{X}_{n}\right) \\ \mathbf{Y}_{s t} & =M\left(\mathbf{X}_{n}\right)\end{aligned}\right.$

Since the solution to (10) is continuous, $P$ is also continuous.

It should be noted that the map $M$ in (10) is a noninvertible map within the whole set of the solution. For example, if $\psi_{1}$ and $\psi_{2}$ are simultaneously nonzero, $\mathbf{X}$ is a multivalued function of $\mathbf{Y}$. However, the map $M$ in (11) is homeomorphism, namely $M$ and $M^{-3}$ are continuous, because one of $\psi_{1}$ and $\psi_{2}$ of $\mathbf{X}_{n}$ is zero. Thus, the orbits $\left\{\mathbf{X}_{n}\right\}$ and $\left\{\mathbf{Y}_{n}\right\}$ are topologically equivalent.

The Jacobian matrix $D P\left(\mathbf{X}_{n}\right)$ of the Poincare map $P$ with respect to $\mathbf{X}_{n}$ is the solution $\mathbf{Z}_{n+1}=\mathbf{Z}\left(\theta_{n+1}\right) \in \mathfrak{R}^{3} \times \Re^{3}$ of the variational equation of its underlying system as given by:

$\dot{\mathbf{Z}}(\theta)=\frac{\partial f_{\theta}(\mathbf{X}(\boldsymbol{\theta}), \theta)}{\partial \mathbf{X}} \mathbf{Z}(\theta), \quad \mathbf{Z}\left(\theta_{n}\right)=\mathbf{Z}_{n}$

Consider that the winding 1 is inactivated, it yields:

$\frac{\partial f_{\theta}}{\partial \mathbf{X}}=\left(\begin{array}{ccc}\frac{-T_{e}+T_{1}}{J \omega^{2}} & \frac{1}{J \omega} \frac{\partial i_{1}}{\partial \theta} & \frac{1}{J \omega} \frac{\partial i_{2}}{\partial \theta} \\ \frac{R i_{1}-u_{1}}{\omega^{2}} & \frac{1}{\omega} \frac{\partial u_{1}}{\partial \psi_{1}}-\frac{R}{\omega} \frac{\partial i_{1}}{\partial \psi_{1}} & 0 \\ \frac{1}{\omega} \frac{\partial u_{2}}{\partial \omega}+\frac{R i_{2}-u_{2}}{\omega^{2}} & 0 & -\frac{R}{\omega} \frac{\partial i_{2}}{\partial \psi_{2}}\end{array}\right)_{(13)}$
$\frac{\partial u_{1}}{\partial \psi_{1}}=-\delta_{\theta}\left(\theta_{\psi}-\theta\right)$
$\frac{\partial u_{2}}{\partial \omega}=\frac{g V_{s} \delta_{\theta}\left(\theta-\theta_{\omega}\right)}{g\left(-B \omega\left(\theta_{\omega}\right)+T_{e}\left(\theta_{\omega}\right)-T_{L}\right) /\left(J \omega\left(\theta_{\omega}\right)\right)-\left(v_{u}-v_{l}\right) / \theta_{T}}$

where $\delta_{\theta}$ is a Dirac delta function that is the derivative of $\varepsilon$. 


\section{Remarks:}

(i). For an arbitrary nonzero point $\mathbf{X}_{n}$, the corresponding initial value $Z_{n}$ is usually an identity matrix. However, since the winding 2 is activated, namely $\psi_{2}=0$, the partial derivative of this zero flux linkage with respect to $\mathbf{X}_{n}$ is also a zero vector. Hence, the third row and column of $\mathbf{Z}_{n}$ are zero vectors. It results that the third column of $Z_{n+1}$ is also a zero vector because the variational equation of each column of $\mathbf{Z}(\theta)$ is linear and homogeneous.

(ii). The rotor displacement $\theta_{\psi}$ of (14) is the position in which $\psi_{1}\left(\theta_{\psi}\right)=0 . \partial u_{1}\left(\theta, \psi_{1}\right) / \partial \psi_{1}$ can be derived from that the partial derivatives of this flux linkage with respect to $\mathbf{X}_{n}$ remain a zero vector after $\theta_{w}$. Therefore, the second row of $\mathbf{Z}_{n+1}$ is always equal to zero.

(iii). The rotor displacement $\theta_{\omega}$ of (15) is the intersection point in which $v_{r}\left(\theta_{\omega}\right)-v_{c}\left(\theta_{\omega}\right)=0$. Due to the $\delta_{\theta}$ pulse in (14) and (15), $\mathbf{Z}(\theta)$ is discontinuous at $\theta_{\varphi}$ and $\theta_{\omega}$. But $Z_{n+\xi}$ is continuous over $Z_{n}$ if $\delta_{\theta}$ is continuous over $\mathbf{Z}_{n}$. However, $\mathbf{Z}_{n+1}$ is discontinuous over $\mathbf{Z}_{n}$ if $\theta_{\omega}$ locates at the changing point between pulsing and skipping cycles, namely $v_{c}\left(\theta_{\omega t}\right)=v_{u}$, where $\partial u_{2}(\theta, \omega) / \partial \omega$ is given by (15) at $\theta_{\omega}^{-}$, whereas $\partial u_{2}(\theta, \omega) / \partial \omega=0$ at $\theta_{\omega}^{+}$. The Poincaré map that its derivative is continuous over distinct regions in the state space but it is discontinuous in the border of these regions is called a piecewise smooth map.

\section{ANALYSIS OF CHAOTIC BEHAVIOR}

Since the Poincaré map and its Jacobian matrix are derived from the numerical solution of the underlying differential equations, the modeling of chaos of the SR motor drive needs to resort to the numerical simulation. A natural numerical tool to obtain the steady-state solution of the Poincare map is the brute-force method - repeating the iteration of the map until the transient has died out or the steady state has been reached [8]. This method has the advantage of simplicity, but may suffer from tedious simulation due to long-live transients. The more efficient approach to obtain the periodic solutions is to locate the corresponding fixed points of the Poincare map by using the Newton-Raphson algorithm. Hence, the stable regions of the system parameters can also be figured out by using this Newton-Raphson algorithm and calculating the corresponding characteristic multipliers that are eigenvalues of the Jacobian matrix. However, the most effective approach to locate chaotic solutions is still the brute-force method. The bifurcation diagrams are also figured out by using the bruteforce method.

\section{A. Fundamental Operation}

Actually, the period of $\psi_{3}$ and $\psi_{2}$ of $\mathbf{X}$ is always $2 \theta_{\text {. }}$ instead of $\theta_{s}$. In order to attain the period-1 orbit of $P$, the winding 1 aiways stands for the inactivated winding at each iteration of $P$, resulting that $\psi_{1}$ and $\psi_{2}$ must exchange their values after each iteration of $P$. Thus, the fixed point $\mathbf{X}^{*}$ of $P$ and its Jacobian matrix are defined as:

$\mathbf{X}^{*}=\mathbf{C P}\left(\mathbf{X}^{*}\right)$

$\mathbf{J}_{1}=\mathbf{C D P}\left(\mathbf{X}^{*}\right)$

where $\mathbf{C}=\left(\begin{array}{lll}1 & 0 & 0 \\ 0 & 0 & 1 \\ 0 & 1 & 0\end{array}\right)$.

The fixed point $\mathbf{X}^{*}$ of $P$ can also be located by using the Newton-Raphson algorithm as given by:

$\mathbf{X}^{(i+1)}=\mathbf{X}^{(i)}-\left(\mathbf{C D} P\left(\mathbf{X}^{(i)}\right)-1\right)^{-1}\left(\mathbf{C} P\left(\mathbf{X}^{(i)}\right)-\mathbf{X}^{(i)}\right)$

where $D P\left(\mathbf{X}^{(i)}\right)$ can be evaluated from (12) and (15).

By checking the characteristic multipliers, the stable regions of the fundamental operation can be figured out. However, It should be noted that the leading magnitude of the characteristic multipliers on the boundary of the stable regions may be not equal to unity, because it may jump from the interior of the unit cycle to the exterior due to the discontinuity of $\mathbf{J}_{1}$.

\section{B. Subharmonics}

The period $-p$ orbit $\left\{\mathbf{X}_{1}^{*}, \cdots, \mathbf{X}_{p}^{*}\right\}$ is defined as:

$$
\mathbf{X}_{k+1}^{*}=\mathbf{C P}\left(\mathbf{X}_{k}^{*}\right) \quad(k=1, \cdots, p-1), \quad \mathbf{X}_{1}^{*}=\mathbf{C P}\left(\mathbf{X}_{p}^{*}\right)
$$

They are a set of fixed points of the $p$-fold iterative map, which can be expressed as:

$\mathbf{X}_{k}^{*}=(\mathbf{C} P)^{(p)}\left(\mathbf{X}_{k}^{*}\right) \quad(k=1, \cdots, p)$

The corresponding Jacobian matrix $\mathbb{J}_{p}$ is given by:

$J_{p}=\operatorname{CDP}\left(\mathbf{X}_{p}^{*}\right) \cdots \operatorname{CDP}\left(\mathbf{X}_{1}^{*}\right)$

The fixed point $\mathbf{X}_{1}^{*}$ can be located by using the NewtonRaphson algorithm given by:

$\mathbf{X}^{(i+1)}=\mathbf{X}^{(i)}-\left(\mathbf{J}_{p}^{(i)}-\mathbf{1}\right)^{-1}\left((\mathbf{C} P)^{(n)}\left(\mathbf{X}^{(i)}\right)-\mathbf{X}^{(i)}\right)$

where:

$\mathbf{J}_{p}^{(i)}=\mathbf{C D P}\left(\mathbf{X}_{p}^{(i)}\right) \cdots \mathbf{C D P}\left(\mathbf{X}_{i}^{(i)}\right)$

According to Remarks (i) and (ii), one of eigenvalues of $J_{1}$ and $J_{p}$ must be zero. Therefore, it can be identified that the three-dimensional Poincare map $P$ is only of the secondorder dynamics. This is also another evidence for two orbits $\left\{\mathbf{X}_{n}\right\}$ and $\left\{\mathbf{Y}_{n}\right\}$ with different dimensionality are topologically equivalent. Nevertheless, in order to analyze the dynamics without rough assumption, the third-dimensional Poincaré map $P$ is still needed. 


\section{COMPUTER SIMULATIONS}

The computer simulations and experimental verifications are carried out by using the 3-phase SR motor drive that is designed for an electric vehicle [9]. The selected motor drive is with the following practical component and parameter values: $N_{s}=12, N_{r}=8, \theta_{d}=\theta_{s}=15^{\circ}, \theta_{o}=3.75^{\circ}, R=$ $0.15 \Omega, B=0.0005 \mathrm{Nm} / \mathrm{rads}^{-1}, J=0.025 \mathrm{kgm}^{2}, V_{s}=150 \mathrm{~V}, v_{u}$ $=5 \mathrm{~V}, v_{l}=1 \mathrm{~V}, \theta_{n}=10, \theta_{i}=1.5^{\circ}, \mathrm{g}=3.5 \mathrm{~V} / \mathrm{rads}^{-1}, \omega_{c}=$ $50 \mathrm{rad} / \mathrm{s}, T_{l}=8 \mathrm{Nm}$.

When $g=1.5 \mathrm{~V} / \mathrm{rads}^{-1}$, the steady-state behavior of the SR motor drive is a fundamental operation. The corresponding waveforms of $v_{c}, v_{r}$ and $i$ as well as the phase-plane trajectory of $i$ versus $\omega$ are shown in Fig. 2, in which $\theta$ is expressed as the integer multiple of the commutation angle $\theta_{s}$. As shown in Fig. 2(a), there is no skipping cycle during PWM regulation, namely $v_{c}$ crosses every $v_{r}$. Since $n_{\theta}=10, i$ has ten peaks within each $\theta_{s}$. Also, the distributions of $i$ are of periodic oscillation as shown in Fig. 2(b). This oscillation includes two components - one corresponds to the commutation frequency and another corresponds to the PWM frequency. Since $\omega$ and $v_{c}$ have the same shape and obey a linear relationship as given by (2), the oscillation of $\omega$, as shown in Fig. 2(a), is mainly of commutation frequency, indicating that the speed oscillation is insensitive to the current component with the PWM frequency. The phase-plane trajectory of this periodic solution is a cycle having ten peaks as shown in Fig. 2(c). The boundary of $\omega$ is from $52.7 \mathrm{rad} / \mathrm{s}$ to $53.0 \mathrm{rad} / \mathrm{s}$, whereas the boundary of $i$ is from $20 \mathrm{~A}$ to $60 \mathrm{~A}$.

When $g=4.4 \mathrm{~V} / \mathrm{rads}^{-1}$, the SR motor drive operates in chaos. The chaotic waveforms and trajectory are shown in Fig. 3. Different from the periodic solution, it has skipping cycles within each $\theta_{s}$ as shown in Fig. 3(a). Furthermore, the number of skipping cycles within each $\theta_{s}$ is a random-like variable. It follows that the oscillating magnitudes of $i$ and $\omega$ are all fluctuating as shown in Figs. 3(a)-(b), respectively. As shown in Fig. 3(c), the trajectory of $i$ versus $\omega$ is a randomlike bounded phase portrait. The boundary of $\omega$ is still acceptable (from $50.7 \mathrm{rad} / \mathrm{s}$ to $51.6 \mathrm{rad} / \mathrm{s}$ ), whereas the boundary of $i$ is exceptionally large (from $0 \mathrm{~A}$ to $120 \mathrm{~A}$ ).

By using the brute-force method to compute the Poincare map (11), the bifurcation diagrams of $i$ and $\omega$ with respect to $g$ and $T_{1}$ are shown in Figs. 4(a)-(c), respectively. They can depict the periodic and chaotic orbits, illustrating how to route to chaos. As shown in Figs. 4(a)-(b), both $i$ and $\omega$ bifurcate from the period-1 orbit to the period-2 orbit at $g=$ $3.92 \mathrm{~V} / \mathrm{rads}^{-1}$, then to the period 4 orbit at $g=4.13 \mathrm{~V} / \mathrm{rads}^{-1}$, then to the period- 8 orbit at $g=4.25 \mathrm{~V} / \mathrm{rads}^{-1}$, and finally route to chaos at $g=4.33 \mathrm{~V} / \mathrm{rads}^{-1}$. As shown in Fig. $4(\mathrm{c}), i$ deviates from the period- 1 orbit to the period-2 orbit at $T_{i}=$ $11 \mathrm{Nm}$, then to the period -4 orbit at $T_{l}=11.4 \mathrm{Nm}$, and finally jump to chaos. Therefore, the route to chaos of the SR motor drive includes not only the period-doubling, but also the abnormality (directly from the period-4 orbit to chaos), the so-called borderline collisions in the piecewise smooth map [10].

By using Newton-Raphson algorithm, the period-1 orbit of $i$ with respect to $g$ is shown in Fig. 5(a), which includes the stable and unstable period-1 orbits. Their characteristic multipliers of $\left|\lambda_{1}\right|$ and $\left|\lambda_{2}\right|$ with respect to $g$ are shown in Figs. $5(\mathrm{~b})$-(c), in which $\left|\lambda_{1}\right|$ is greater than unity when $g>$ $3.92 \mathrm{~V} / \mathrm{rads}^{-1}$. It indicates that the period-1 orbit is unstable when $g>3.92 \mathrm{~V} / \mathrm{rads}^{-1}$. Similarly, the period-2 orbit of $i$ with respect to $g$ is shown in Fig. 6(a), which includes the stable period-1 orbit as well as stable and unstable period- 2 orbits. Their characteristic multipliers of $\left|\lambda_{1}\right|$ and $\left|\lambda_{2}\right|$ with respect to $g$ are shown in Figs. $6(\mathrm{~b})-(\mathrm{c})$, in which $\left|\lambda_{1}\right|$ is greater than unity when $g>4.13 \mathrm{~V} / \mathrm{rads}^{-1}$. It indicates that the period-2 orbit is unstable when $g>4.13 \mathrm{~V} / \mathrm{rads}^{-1}$. It should be noted that the above results using Newton-Raphson algorithm agree closely with the bifurcation diagrams using the bruteforce method as given by Fig. 4. The Newton-Raphson algorithm facilitates the identification of the desired stable operating regions for different system parameters and conditions.

From bifurcation diagrams as shown in Figs. 5-6, it can be concluded that the Poincare map of the SR motor drive has two key features. One is that its Jacobian matrix and hence characteristic multipliers are discontinuous over the system parameters even though the map is continuous over the system parameters, namely the Poincare map is a piecewise smooth map. In this kind of maps, the bifurcation point occurs at not only the points whose characteristic multipliers locate in the unity cycle, but also the discontinuity points whose characteristic multipliers jump from the interior of the unit cycle to its exterior. The second feature is that one of characteristic multipliers is much smaller than the other one if they are both real numbers. Moreover, for the unstable periodic orbit, this small characteristic multiplier is nearly zero, resulting that chaotic behavior will has very strong phase space contraction in this direction.

\section{EXPERIMENTAL VERIFICATIONS}

For the sake of simplicity and clarity, the speed feedback control voltage $v_{c}$ is measured instead of the actual motor speed. The measured trajectory and waveforms of $i$ and $v_{\varepsilon}$ : with $g=1.5 \mathrm{~V} / \mathrm{rads}^{-1}$ are shown in Fig. 7. This illustrates that the SR motor drive operates in the period-1 orbit. Also, it can be found that $i$ lies roughly between $20 \mathrm{~A}$ and $60 \mathrm{~A}$ while $v_{c}$. lies between $4 \mathrm{~V}$ and $4.5 \mathrm{~V}$ (equivalent to $\omega$ between $52.6 \mathrm{rad} / \mathrm{s}$ and $53 \mathrm{rad} / \mathrm{s}$ ). By comparing these results with the waveforms and trajectory shown in Fig. 2, the measured results and the theoretical prediction have a good agreement. 


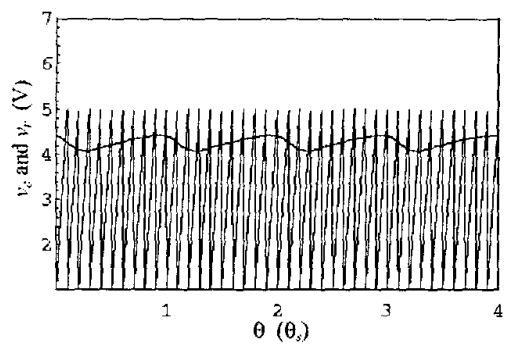

(a)

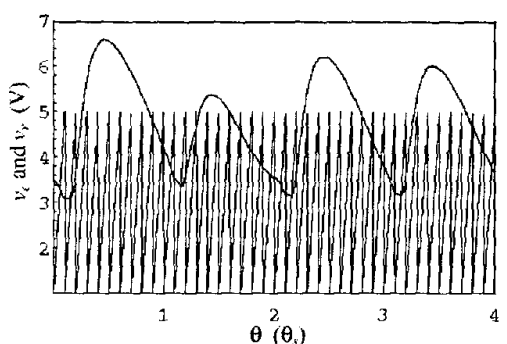

(a)

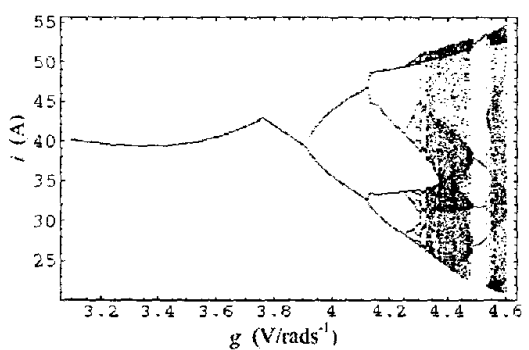

(a)

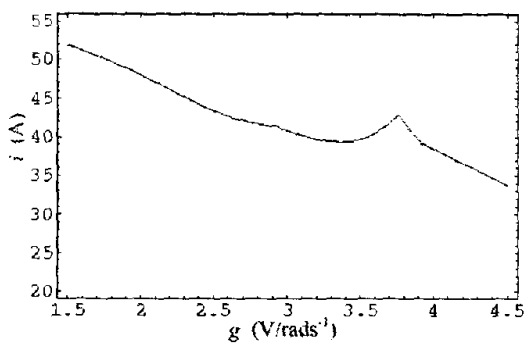

(a)

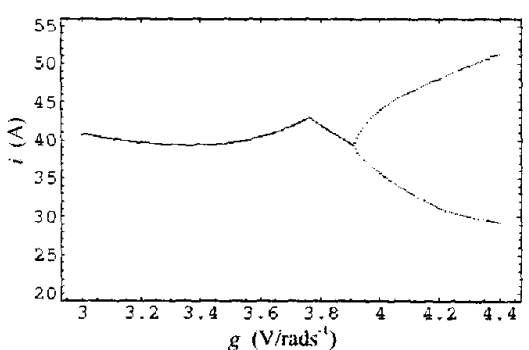

(a)

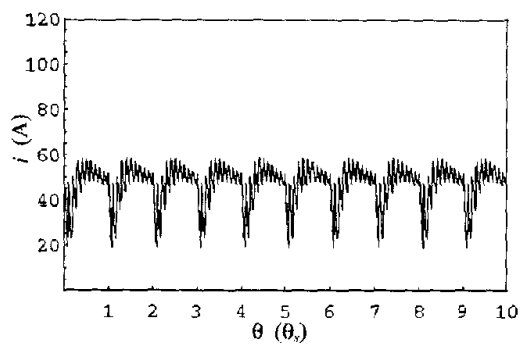

(b)

Fig. 2. Fundamental operation.

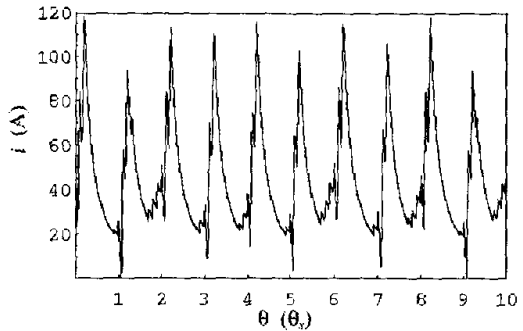

(b)

Fig. 3. Chaotic behavior.

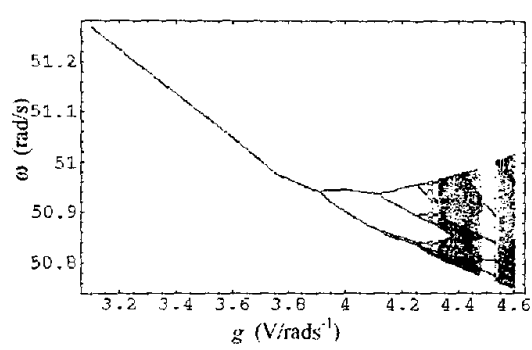

(b)

Fig. 4. Bifurcation diagrams.

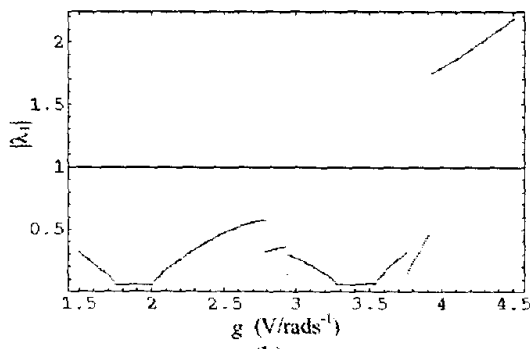

(b)

Fig. 5. Period-1 operation.

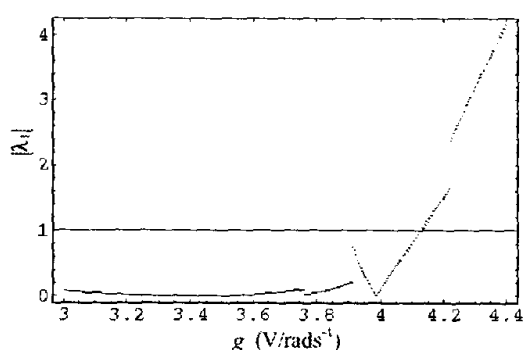

(b)

Fig. 6. Period-2 operation.

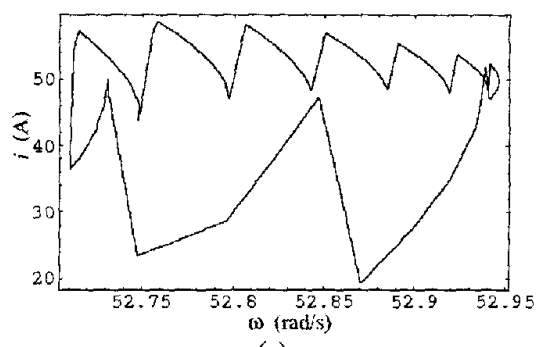

(c)

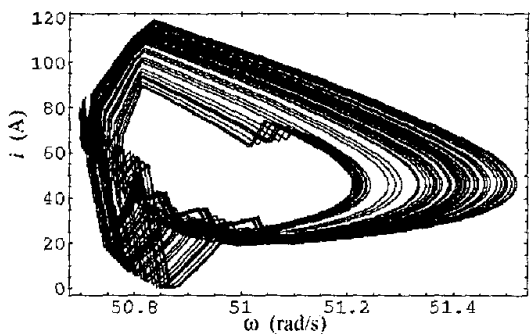

(c)

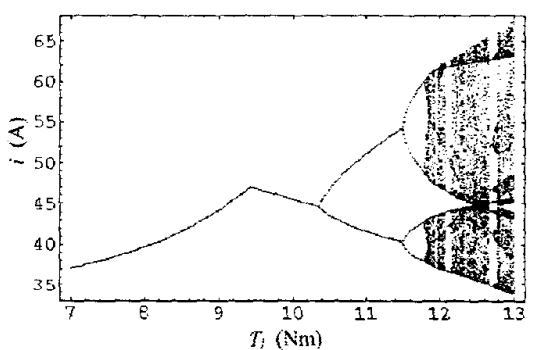

(c)

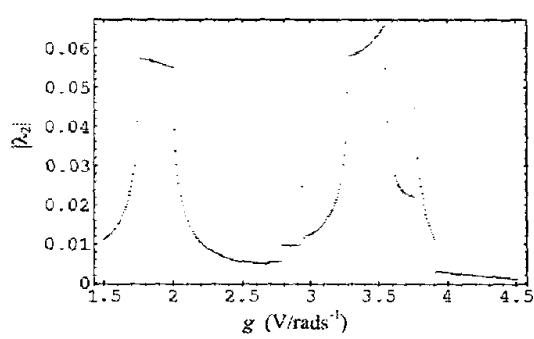

(c)

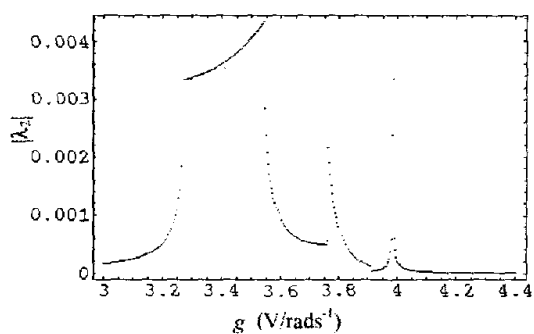

(c) 
Moreover, by selecting $g=4.4 \mathrm{~V} / \mathrm{rads}^{-1}$, the measured trajectory and waveforms of $i$ and $v_{i}$, as shown in Fig. 8, illustrate that the SR motor drive operates in chaos. It can be found that the boundaries of $i$ and $v_{c}$ lie roughly between $0 \mathrm{~A}$ and $120 \mathrm{~A}$ and between $2 \mathrm{~V}$ and $8 \mathrm{~V}$ (equivalent to $\omega$ between $50.45 \mathrm{rad} / \mathrm{s}$ and $51.8 \mathrm{rad} / \mathrm{s}$ ), respectively. Different to the period-1 orbit in which the measured trajectory and waveforms are directly compared with the theoretical prediction, the chaotic trajectory and waveforms measured in the experiment can hardly match with the theoretical ones because the chaotic behavior is not periodic such that the period of measurement can not be the same with that of theoretical analysis. Also, its characteristics are extremely sensitive to the system initial conditions. Nevertheless, it can be found that the measured boundaries of the chaotic trajectory shown in Fig. 8 resemble to the theoretical prediction in Fig. 3.

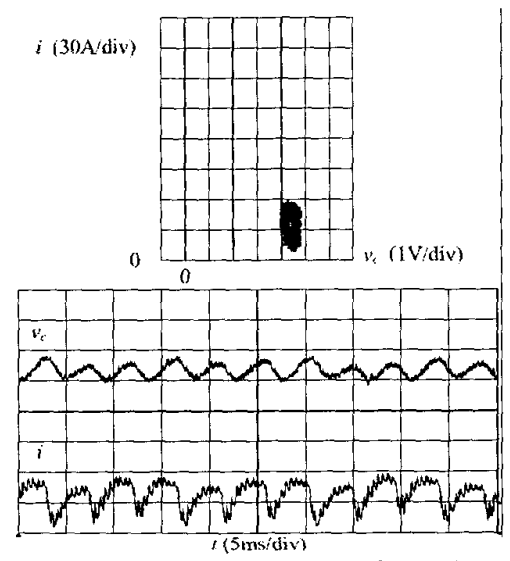

Fig. 7. Measured trajectory and waveforms of feedback control voltage and total current during fundamental operation.

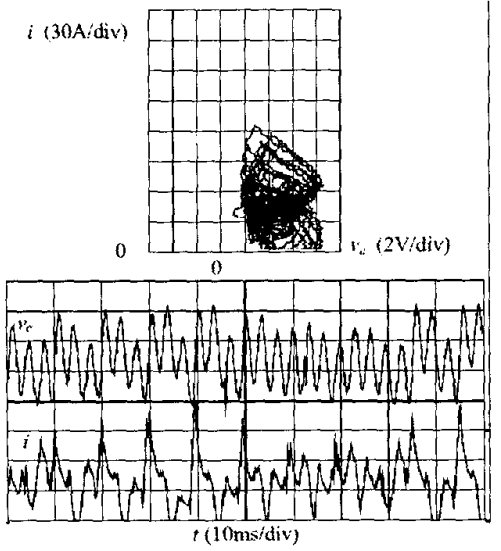

Fig. 8. Measured trajectory and waveforms of feedback control voltage and total current during chaotic operation.

\section{CONCLUSION}

In this paper, the modeling and analysis of chaotic behavior in SR motor drives using the voltage PWM regulation is proposed. By selecting the flux linkage and rotor position as the state variable and independent variable, respectively, a Poincaré map and its Jacobian matrix are constructed, which take the advantages to significantly reduce the computation time to analyze the periodic and chaotic behavior. The bifurcation diagrams illustrate that the route to chaos of the SR motor drive includes not only the period-doubling, but also the abnormality (directly from the period-4 orbit to chaos). The theoretical modeling and analysis are verified by using both computer simulations and experimental measurements. The proposed approach and derived modeling can readily be applied or extended to other SR motor drives.

\section{ACKNOWLEDGMENT}

This work was supported by a grant from the Research Grants Council of Hong Kong Special Administrative Region, China (Project No. HKU 7128/99E).

\section{REFERENCES}

[1] P.J. Lawrenson, "A brief status review of switched reluctance drives." EPE Journal, Vol. 2. No. 3, 1992, pp.133-144.

[2] K.T. Chau, J.H. Chen, C.C. Chan, and Q. Jiang, "Subharmonics and chaos in switched reluctance motor drives," Proceedings of IEEE International Electric Machines and Drives Conference, 1999, pp.661-663.

[3] J.R. Wood. "Chaos: a real phenomenon in power electronics." Proceedings of IEEE Applied Power Electronic: Conference, 1989, pp. 115-123.

[4] D.C. Hamill, J.H.B. Deane, and D.J. Jefferies, "Modeling of chaotic dc-dc converters by iterated nonlinear mappings," IEEE Transactions on Power Electronics, Vol. 7, No. 1, 1992, pp.25-36.

[5] I. Nagy, L. Matakas Jr. and E. Masada, "Application of the theory of chaos in PWM technique of induction motors." Proceedings of International Power Electronics Conference, 1995, pp.58-63.

[6] N. Hemati, "Strange attractors in brushless DC motors," IEEE Transactions on Circuits and Systems-l: Fundamental Theory and Applications, Vol. 41, №. 1, 1994, pp.40-45.

[7] J.H. Chen, K.T. Chau, and C.C. Chan, "Chaos in voltage-mode controlled de drive system," International Journol of Electronics, Vol. 86, No. 7, 1999, pp. 857-874.

[8] T.S. Parker and L.O. Chua, Practical Numerical Algorithms for Chaoric Systems. Springer-Verlag. 1989.

[9] C.C. Chan, Y. Zhan, Q. Jiang, and K.T. Chau, "A high performance switched reluctance motor drive for P-star EV project," Proceedings of International Electric Vehicle Symposium, 1996, pp.78-83.

[10] G. Yuan, S. Banerjee, E. Ott, and 3.A. Yorke, "Border-collision bifurcation in the buck converter," IEEE Transactions on Circuits and Systems-1: Fundamental Theory and Applications, Vol. 45, No. 7, 1998, pp. $707-716$ 